\section{Cistoadenocarcinoma mucinoso de apéndice: diagnóstico endoscópico}

Palabras clave: Tumor de apéndice. Adenocarcinoma. Tumor mucinoso. Pseudomixoma peritoneal. Cistoadenocarcinoma mucinoso.

Key words: Appendix tumor. Adenocarcinoma. Mucinous tumor. Pseudomyxoma peritonei. Mucinous cystoadenocarcino$m a$.

\section{Sr. Director:}

Los tumores primarios de apéndice son diagnosticados en el $0,9-1,4 \%$ de las piezas de apendicectomía (1). El tumor primario de apéndice fue descrito inicialmente en 1903, en una serie de casos publicada por Elting (1). Presentamos el caso de una paciente diagnosticada de un tumor apendicular de forma preoperatoria y confirmado con la cirugía y estudio histológico posterior.

\section{Caso clínico}

Mujer de 69 años con alergia a la penicilina, HTA en tratamiento con valsartán, dislipemia tratada con atorvastatina, que consultó en cirugía por rectorragias ocasionales no mezcladas con las heces desde hacía varios años. En la ileocolonoscopia (Figs. 1 y 2) se observaron hemorroides externas y una tumoración en el interior del apéndice con úlcera excavada en su seno, cuyas biopsias fueron informadas como sin alteraciones relevantes. Con el diagnóstico de tumor apendicular se intervino a los 24 días hallando tumor en la base apendicular de aspecto mucoide que infiltraba peritoneo, adherido a la trompa, ovario y uréter derecho. Se efectuó hemicolectomía derecha con anastomosis ileocólica y colocación de drenaje. El diagnóstico histológico fue de adenocarcinoma mucinoso (Fig. 3) de apéndice de $4,5 \mathrm{~cm}$ en estadio II.

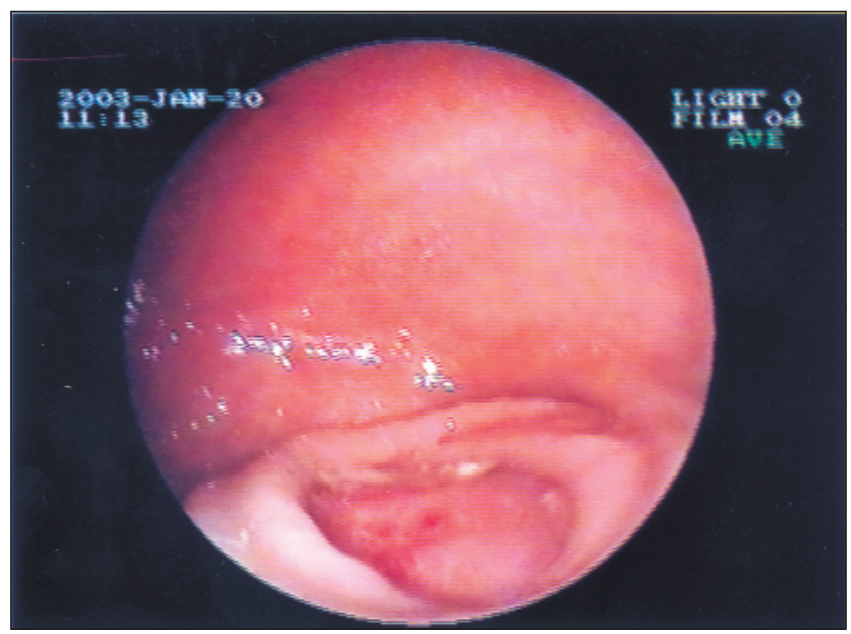

Fig. 1.

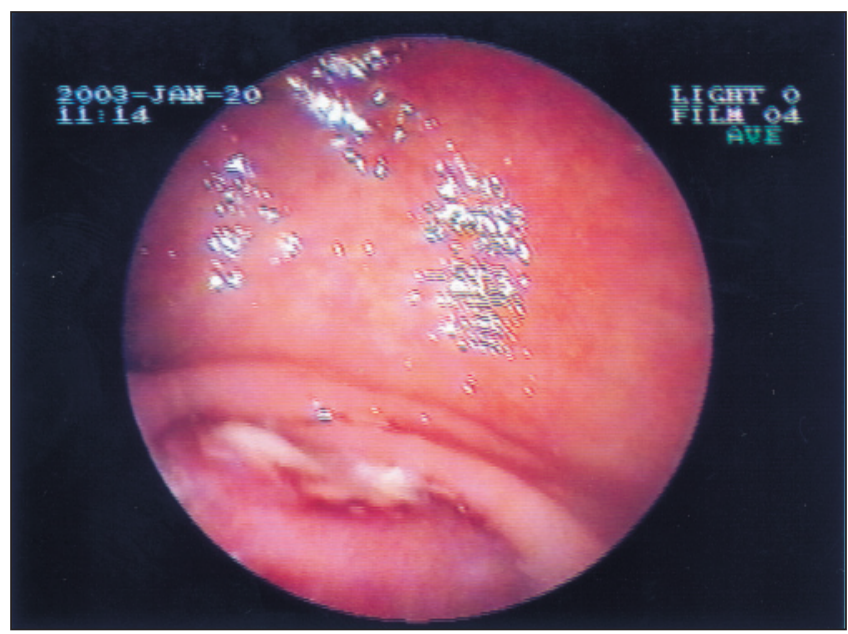

Fig. 2.

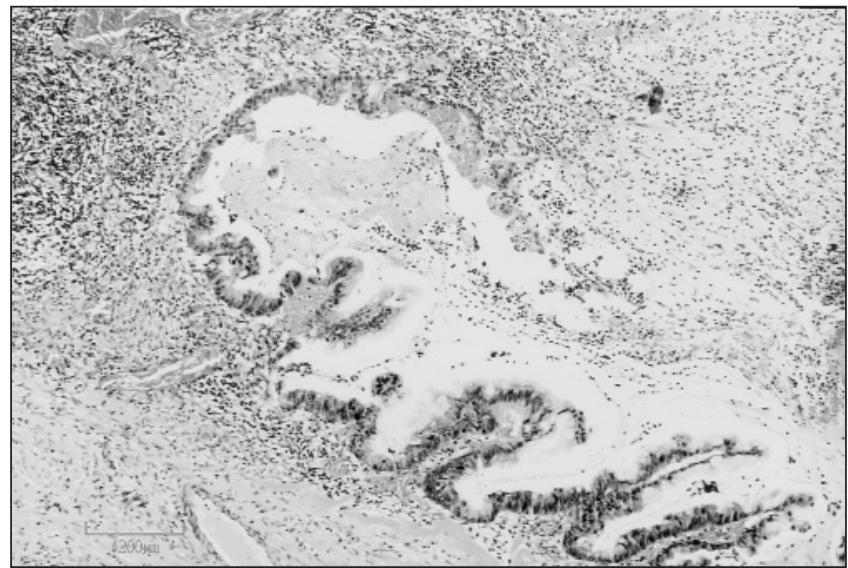

Fig. 3 .

Posteriormente inició tratamiento con 5-fluoracilo/ácido folínico y requirió ingreso al presentar mucositis tras la quimioterapia que se resolvió con el tratamiento habitual.

\section{Discusión}

El adenocarcinoma (AC) primario de apéndice es una neoplasia poco frecuente y representa menos del $0,5 \%$ de los tumores gastrointestinales (2). Según la Clasificación Internacional de Enfermedades Oncológicas, los tumores apendiculares se clasifican en 5 categorías: AC tipo colónico, AC tipo mucinoso, carcinoma de células en anillo de sello, adenocarcinoides y carcinoides (1). En una serie de 1.645 pacientes con tumores apendiculares malignos el AC mucinoso fue el más frecuente de los 5 tipos (37\%), con una incidencia similar en hombres y mujeres y una edad media de 60 años (1).

La forma más común de presentación es como apendicitis aguda, dolor abdominal, masa palpable o ascitis (pseudomixoma peritoneal). Sin embargo, otras veces el diagnóstico se realiza en pacientes asintomáticos de forma incidental en el transcurso de cirugía, exámenes radiológicos o endoscópicos (3), como posiblemente sea nuestro caso, ya que es poco probable que los episodios 
de rectorragia estuvieran relacionados con el tumor por sus características y el largo tiempo de evolución. El diagnóstico preoperatorio es poco frecuente y menos de la mitad de los casos son diagnosticados de forma intraoperatoria (4). La perforación de estos tumores suele originar un pseudomixoma peritoneal y en el caso de mujeres con pseudomixoma se debe excluir un posible tumor ovárico como causa. Pueden coexistir tumores sincrónicos de apéndice y ovario con pseudomixoma peritoneal, surgiendo la duda sobre el posible origen de los mismos. Es importante diferenciar el origen tumoral porque tanto el tratamiento como el pronóstico dependen del tipo de tumor (2).

El tratamiento de elección es la hemicolectomía derecha. Se ha señalado que el tratamiento adyuvante con radioterapia o quimioterapia mejora la supervivenvia (5). La resección tumoral por vía laparoscópica puede asociarse a diseminación peritoneal por lo que debe evitarse (6).

P. A. Rivera Vaquerizo, C. Albaladejo Ortiz' ${ }^{1}$, M. Blasco Colmenarejo, M. Vicente Gutiérrez, J. Mayor López y R. Pérez-Flores
Servicio de Aparato Digestivo ${ }^{\prime}$ Medicina Interna. Complejo Hospitalario Universitario. Albacete

\section{Bibliografiía}

1. McCusker ME, Coté TR, Clegg LX, Sobin LH. Primary malignant neoplasms of the appendix: a population-study from the surveillance, epidemiology and end-results program 1973-1998. Cancer 2002; 94 (12): 3307-12.

2. Ciriza C, Valerdiz S, Toribio C, Dajil S, Romero MJ, Urquiza O, et al. Adenocarcinoma mucinoso de apéndice asociado con tumores ováricos y pseudomixoma peritonei. Dificultad en el diagnóstico diferencial. An Med Interna 2000; 17 (10): 540-2.

3. Sisk CM, Watkins KT, Hayashi PH. Image of the month: appendiceal mucinous tumor. Gastroenterology 2002; 122 (5): 1200, 1548.

4. Ochando F, Landa I, Garmendia C, Vaillo A, Moreno E. Cistoadenocarcinoma mucinoso de apéndice fistulizado a recto. An Med Interna 1999; 16 (12): 657-8.

5. Proulx G, Willett C, Daley W, Shellito P. Appendiceal carcinoma: patterns of failure following surgery and implications for adjuvant therapy. J Surg Oncol 1997; 66: 51-3.

6. González S, Shmookler BM, Sugarbaker PH. Appendiceal mucocele. Contraindication to laparoscopic appendectomy. Surg Endosc 1998; 12 (9): 1177-9. 